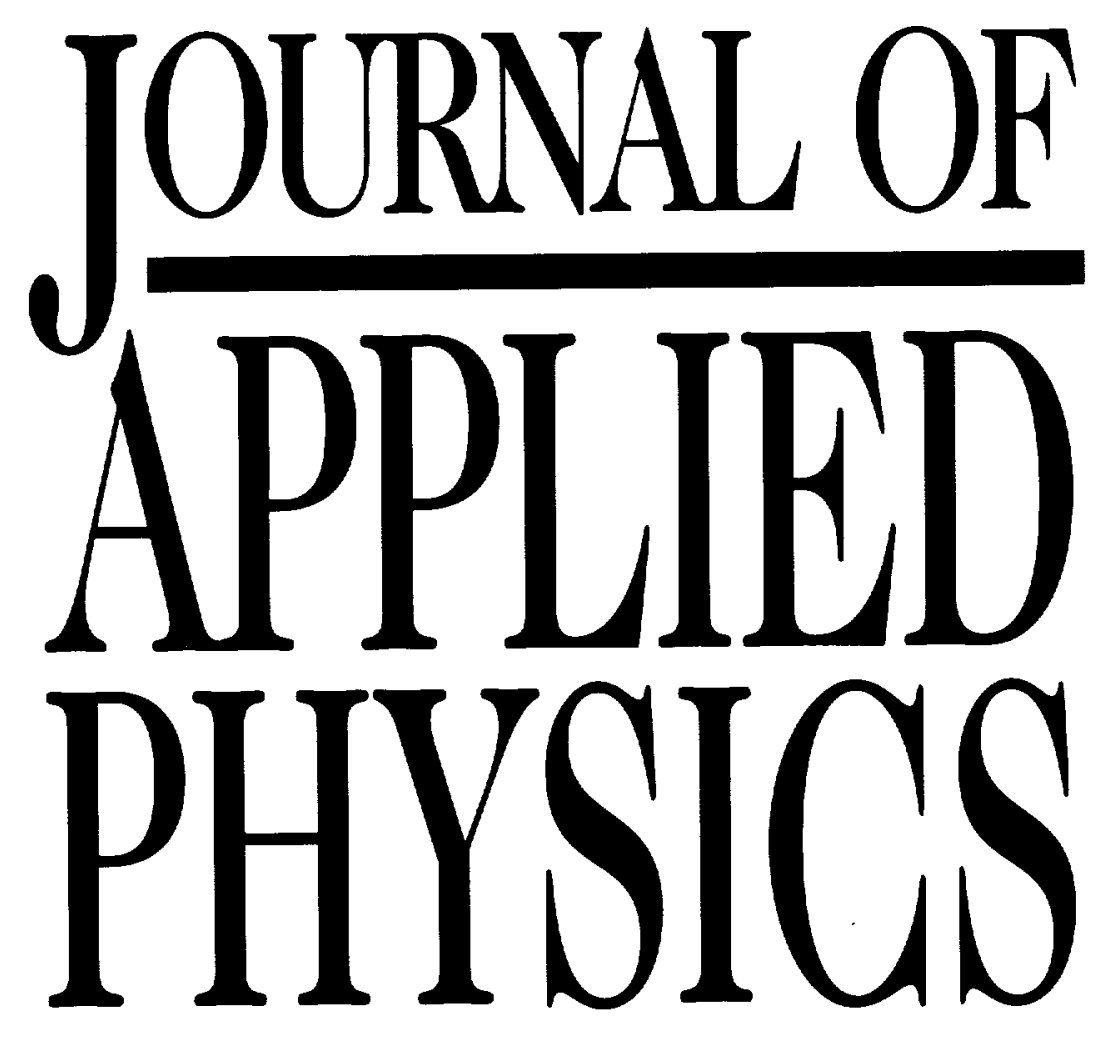

Vol. 81 , No. 5, 1 March 1997

Intrinsic carrier concentration and electron effective mass in $\mathrm{Hg}_{1-x} \mathrm{Zn}_{x} \mathrm{Te}$

Yi-Gao Sha, Ching-Hua Su, and S. L. Lehoczky

Space Science Laboratory, NASA Marshall Space Flight Center Huntsville, Alabama 35812

pp. 2245-2249 



\title{
Intrinsic carrier concentration and electron effective mass in $\mathrm{Hg}_{1-x} \mathbf{Z n}_{x} \mathrm{Te}$
}

\author{
Yi-Gao Sha, ${ }^{\text {a) }}$ Ching-Hua Su, and S. L. Lehoczky \\ Space Science Laboratory, NASA Marshall Space Flight Center Huntsville. Alabuma 35812
}

(Received 13 September 1996; accepted for publication 22 November 1996)

The intrinsic carrier concentrations, Fermi energies, and the electron effective masses are calculated for $\mathrm{Hg}_{1-x} \mathrm{Zn}_{x}$ Te with $0<x \leqslant 0.4$ and $50 \mathrm{~K} \leqslant T \leqslant 400 \mathrm{~K}$. The numerical calculations are based on the Kane $\mathbf{k} \cdot \mathbf{p}$ model and no further analytical simplification or approximation is made for the energy band structure beyond those inherent in the Kane model. The results are compared to the previous calculations. (1) 1997 American Institute of Physics. [S(0)21-8979(97)(040)5-X]

\section{INTRODUCTION}

For over a decade, $\mathrm{HgZnTe}$ has been considered as a potentially superior infrared detector material due to its high bond strength and material stability compared to HgCdTe. ${ }^{1-6}$ A major effort is being devoted to the growth of bulk crystals and epitaxial layers of $\mathrm{HgZnTe}$ as well as their characterizations. ${ }^{7-1.3}$ Recent results on the HgZnTe devices confirm the advantages predicted by theory of $\mathrm{HgZnTe}$ over HgCdTe. ${ }^{14.15}$

Intrinsic carrier concentration is a quantity of fundamental importance to a wide range of solid-state material and device applications. A precise knowledge of the intrinsic carrier concentration should benefit in understanding the performance of $\mathrm{HgZnTe}$ detectors. The earliest calculation of the intrinsic carrier concentration in $\mathrm{Hg}_{1-x} \mathrm{Zn}_{x} \mathrm{Te}$ was carried out by Shneider et al. ${ }^{16}$ who used an energy gap relation, $E_{g}(x, T)$, as a linear function of alloy composition. In contrast to it, Dziuba et al. ${ }^{17}$ described a nonlinear dependence of the energy gap on composition from their optical and electrical measurements. Recently, more accurate expressions for $E_{q}(x, T)$ have been reported by Sher et al. ${ }^{11}$ and Toulouse et al. ${ }^{18}$ both from infrared transmission measurements and the latter has been used by Józwikowski and Rogalski ${ }^{19}$ in the calculation of the intrinsic carrier concentrations and the electron effective masses in $\mathrm{Hg}_{1-\gamma} \mathrm{Zn}_{x} \mathrm{Te}$. However, this particular calculation was based on an approximated solution of the well known Kane $\mathbf{k} \cdot \mathbf{p}$ mode $^{20}$ for the case of $E_{k} \ll \Delta$, where $\Delta$ is the spin-orbit splitting, which is applicable only for the narrow band gap materials such as HgSeTe. (For $\mathrm{Hg}_{1-x} \mathrm{Zn}_{x}$ Te, e.g., for $x=0.2$ and $T=300 \mathrm{~K}$, $E_{\mathrm{p}} \equiv 0.3 \mathrm{eV}$. compared to $\Delta \cong 1.0 \mathrm{eV}$.) The work ${ }^{19}$ subsequently adopted an expression given by Harman and Strauss ${ }^{21}$ for calculating the electron concentration in which the free-electron (parabolic) term in the energy dispersion relation was neglected. For the calculation of the electron effective mass, Józwikowski and Rogalski adopted a relation which was derived by Wright et al. ${ }^{22}$ using a series of approximations including the one for sharp degeneracy which ignores the statistical distribution of the electrons. The authors presented results for two different electron effective masses without appropriate interpretations. Given the restrictive nature of the approximations in their calculations and the

\footnotetext{
a) Universities Space Research Association. Current address: Digirad Corporation, 7408 Trade St. San Diego, CA 92121-2410.
}

ambiguities in the differentiation between the two electron effective masses, we felt another more exact and more specific calculation of these properties was merited.

In this work, the intrinsic carrier concentration and electron effective mass in $\mathrm{Hg}_{1-} \mathrm{Zn}_{\boldsymbol{\gamma}} \mathrm{Te}$ were numerically calculated. We adopt the procedures similar to those used by Su et al. for calculating the intrinsic carrier concentrations in $\mathrm{Hg}_{1 \ldots x} \mathrm{Cd}_{x} \mathrm{Te}^{23}$ which solve the exact dispersion relation in Kane model for the calculation of the conduction band electron concentrations and the corresponding electron effective masses. No approximation beyond those inherent in the $\mathbf{k} \cdot \mathbf{p}$ model was used here.

\section{THEORY AND CALCULATIONS}

The nonparabolicity in the dispersion relation of the energy bands in the Kane $\mathbf{k} \cdot \mathbf{p}$ theory arises from the secular equation describing the conduction, light-mass and split-off valence bands,

$$
\varepsilon\left(\varepsilon+E_{g}\right)\left(\varepsilon+E_{g}+\Delta\right)-k^{2} P^{2}\left(\varepsilon+E_{g}+\frac{2 \Delta}{3}\right)=0,
$$

where $\Delta$ and $P$, the momentum matrix element, are assumed constant throughout the $\mathrm{Hg}_{1-}, \mathrm{Zn}_{x} \mathrm{Te}$ alloy system with the only variable being $E_{q}$, and the zero of energy, $\varepsilon$, is taken at the bottom of the conduction band. For the conduction band. the energy dispersion relation is written as

$$
E=\frac{\hbar^{2} k^{2}}{2 m}+\varepsilon_{r},
$$

where $m$ is the free electron mass and, for positive $E_{s}^{-}, \varepsilon_{c}$ is the largest root of Eq. (1). The density of states in the conduction band is then given by

$$
N_{c}(E)=\frac{k}{\pi^{2}}\left(\frac{\hbar^{2}}{m}+\frac{1}{k} \frac{\partial \varepsilon_{i}}{\partial k}\right)^{-1}
$$

Since there is no identical root for Eq. (1). it can be shown that $\partial \varepsilon_{t} / \partial k$ can be obtained directly from Eq. (1) by taking derivative $\partial \varepsilon / \partial k$ and replacing $\varepsilon$ by $\varepsilon_{\text {, }}$. Thus. 


$$
N_{c}(E)=\frac{\sqrt{U\left(\varepsilon_{c}\right)}}{\pi^{2} P}\left(\frac{\hbar^{2}}{m}+\frac{2 P^{2}}{Z\left(\varepsilon_{c}\right)}\left(\varepsilon_{c}+E_{g}+\frac{2 \Delta}{3}\right)\right)^{-1},
$$

$Z\left(\varepsilon_{c}\right) \equiv\left(\varepsilon_{c}+E_{g}\right)\left(2 \varepsilon_{c}+E_{g}+\Delta\right)+\varepsilon_{c}\left(\varepsilon_{c}+E_{g}+\Delta\right)-U\left(\varepsilon_{c}\right)$,

where

$$
U\left(\varepsilon_{c}\right) \equiv \frac{\varepsilon_{c}\left(\varepsilon_{c}+E_{g}\right)\left(\varepsilon_{c}+E_{g}+\Delta\right)}{\varepsilon_{c}+E_{g}+\frac{2 \Delta}{3}},
$$

and Eq. (1) has been used to eliminate $k$.

The electron concentration in the conduction band is calculated from

$$
n=\int_{0}^{\infty} \frac{N_{c}(E) d E}{1+\exp \left(\frac{E-E_{f}}{k T}\right)}=\int_{0}^{\infty} \frac{\sqrt{U(\varepsilon)} \frac{d}{d \varepsilon} E(\varepsilon)}{\pi^{2} P\left(\frac{\hbar^{2}}{m}+\frac{2 P^{2}}{Z(\varepsilon)}\left(\varepsilon+E_{g}+\frac{2 \Delta}{3}\right)\right)\left[1+\exp \left(\frac{E(\varepsilon)-E_{f}}{k T}\right)\right]} d \varepsilon,
$$

where the subscript $c$ of $\varepsilon$ has been dropped. With Eqs. (1), (2), (5), and (6), integration (7) can be performed without any approximation.

Since the contribution of the nonparabolic light-mass valence band to the hole concentration is at least two orders of magnitude less than that of the parabolic heavy-mass valence band, ${ }^{24}$ only the latter is used to calculate the valence band hole concentration. Thus, we have

$$
p=4 \pi\left(\frac{2 m_{h}^{*} k T}{h^{2}}\right)^{3 / 2} F_{1 / 2}\left(\frac{-E_{f}-E_{g}}{k T}\right),
$$

where $F_{1 / 2}(x)$ is the Fermi-Dirac function.

The energy gap in $\mathrm{eV}$ for $\mathrm{Hg}_{1-x} \mathrm{Zn}_{x} \mathrm{Te}$ as a function of composition and temperature given by Toulouse et al., ${ }^{18}$

$$
\begin{aligned}
E_{g}(x, T)= & -0.3+3.24 \times 10^{-2} \sqrt{x}+2.731 x-0.629 x^{2} \\
& +0.533 x^{3}+5.3 \times 10^{-4} \\
& \times(1-0.76 \sqrt{x}-1.29 x) T,
\end{aligned}
$$

was used for the calculations. A similar relation given by Sher et al. ${ }^{11}$ shows a weaker composition dependence and gives a value of $E_{g}(0.4,300 \mathrm{~K})$ about $13 \%$ lower than that by Eq. (9) while coincides with the latter at $x=0.095$ and $T=300 \mathrm{~K}$.

For negative $E_{g}$, i.e., semimetal, $E_{g}+\Delta$ and $E_{g}+2 \Delta / 3$ should be replaced by $\Delta$ and $2 \Delta / 3$, respectively, ${ }^{25}$ and $E_{g}$ by $\left|E_{g}\right|$ in Eqs. (1), (5), (6), and (7) and $E_{g}$ in Eq. (8) should be replaced by zero.

\section{INTRINSIC FERMI ENERGY AND CARRIER CONCENTRATION}

The intrinsic Fermi energy, $E_{f}^{i}$ can be solved numerically by equating $n$ given by Eq. (7) and $p$ by Eq. (8). Using the calculated $E_{f}^{i}$, either Eq. (7) or (8) can be used to calculate the intrinsic carrier concentration, $n_{i}$. Same band parameters were assumed as those used in the recent calculation for electron mobilities in $\mathrm{Hg}_{1-x} \mathrm{Zn}_{x} \mathrm{Te}^{24,26} \Delta=0.95 \mathrm{eV}$, $P=8.2 \times 10^{-8} \mathrm{eV} \mathrm{cm}$, and $m_{h}^{*}=0.6 \mathrm{~m}$, where the value for $\Delta$ is the average of those found in literature. ${ }^{19,27}$ During the calculation, the upper limit of $\varepsilon$ in the integral (7) was se- lected for each $x$ and $T$ such that changing to a higher value did not essentially affect the calculated result and that the integrand, at this upper limit, had decreased to less than one half of one thousandth of its maximum. This upper limit corresponds to a conduction band energy that ranges from 11 $\mathrm{kT}$ for $x=0.1$ at $400 \mathrm{~K}$ to $97 \mathrm{kT}$ for $x=0.4$ at $50 \mathrm{~K}$ above the calculated $E_{f}^{i}(x, T)$. The $E_{f}^{i}$ was considered to be solved when $|n / p-1|<10^{-4}$ was satisfied. The calculated intrinsic carrier concentrations in semiconductor $\mathrm{Hg}_{1-x} \mathrm{Zn}_{x} \mathrm{Te}$ as a function of temperature for various compositions in the range $0<x \leqslant 0.4$ are plotted in Fig. 1 . The differences in the

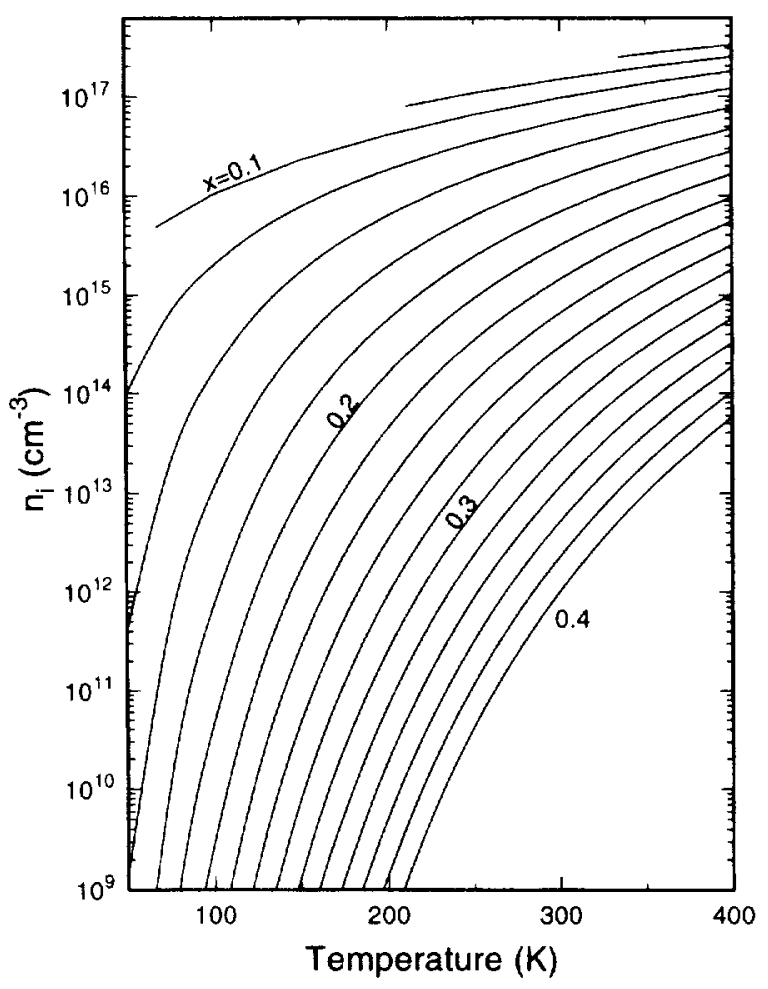

FIG. 1. Calculated intrinsic carrier concentrations in $\mathrm{Hg}_{1-x} \mathrm{Zn}_{x} \mathrm{Te}$ as functions of temperature. The $x$ value is 0.06 for the upper most curve and, with a 0.02 interval, in an ascending order for the other curves from the top to bottom, as indicated by the labels. 
TABLE l. Differences in the intrinsic carrier concentrations between the current and previous calculations.

\begin{tabular}{|c|c|c|c|c|c|c|c|c|c|c|c|c|c|c|c|}
\hline \multirow{2}{*}{$\frac{T}{x}$} & \multicolumn{3}{|c|}{$350 \mathrm{~K}$} & \multicolumn{3}{|c|}{$300 \mathrm{~K}$} & \multicolumn{3}{|c|}{$250 \mathrm{~K}$} & \multicolumn{3}{|c|}{$2(k) \mathrm{K}$} & \multicolumn{3}{|c|}{$150 \mathrm{~K}$} \\
\hline & This work & Ref. 19 & $\%$ & This work & Ref. 19 & $\%$ & This work & Ref. 19 & $\%$ & This work & Ref. 19 & $\%$ & This work & Ref. 19 & $y$ \\
\hline 0.1 & $1.33 \times 10^{17}$ & $1.21 \times 10^{17}$ & 10 & $9.65 \times 10^{16}$ & $8.75 \times 10^{16}$ & 10 & $6.60 \times 10^{16}$ & $5.86 \times 10^{16}$ & 13 & $4.16 \times 10^{16}$ & $3.48 \times 10^{16}$ & 20 & $2.31 \times 10^{i t}$ & $1.95 \times 10^{16}$ & 18 \\
\hline 0.2 & $8.00 \times 10^{15}$ & $6.96 \times 10^{15}$ & 15 & $3.16 \times 10^{15}$ & $2.76 \times 10^{15}$ & 14 & $9.18 \times 100^{14}$ & $8.11 \times 10^{14}$ & 13 & $1.57 \times 10^{14}$ & $1.37 \times 10^{14}$ & 15 & $9.59 \times 10^{12}$ & $7.30 \times 10^{12}$ & 31 \\
\hline 0.3 & $2.96 \times 10^{14}$ & $2.60 \times 10^{14}$ & 14 & $5.88 \times 10^{13}$ & $5.21 \times 10^{1.3}$ & 13 & $6.49 \times 10^{12}$ & $6.15 \times 10^{12}$ & 6 & $2.59 \times 10^{11}$ & $2.23 \times 10^{11}$ & 16 & $1.38 \times 100^{9}$ & $1.10 \times 101^{13}$ & 25 \\
\hline 0.4 & $1.01 \times 10^{17}$ & $9.22 \times 10^{12}$ & 10 & $1.01 \times 10^{12}$ & $9.12 \times 10^{11}$ & 11 & $4.25 \times 10^{10}$ & $3.97 \times 10^{10}$ & 7 & & & & & & \\
\hline
\end{tabular}

intrinsic carrier concentrations between the present calculation and that in Ref. 19 at several $x$ and $T$ values are listed in Table I. The numerical data for Ref. 19 were scaled from the plotted curves. The previous results are consistently lower by $10 \%-15 \%$ and more so at lower temperatures. Even when using exactly the same values for band parameters $\Delta$ and $P$ as in Ref. 19, the present results are still about $7 \%-25 \%$ higher than those previously reported. The temperature dependence of the reduced Fermi energy, $E_{f}^{i} / k T$, for the intrinsic semiconductor material is shown in Fig. 2. The figure indicates that intrinsic $\mathrm{Hg}_{1-x} \mathrm{Zn}_{x}$ Te remains nondegenerate for $x$ value greater than about 0.2 for the entire temperature range.

\section{NEAR THE BAND CROSS-OVER}

As suggested by Eq. (9), the energy band gap in $\mathrm{Hg}_{1-{ }_{x}} \mathrm{Zn}_{8} \mathrm{Te}$ decreases with temperature and approaches zero at 334,211 , and $68 \mathrm{~K}$ for $x=0.06,0.08$, and 0.1 , respectively. Below these temperatures, the band gaps, defined as

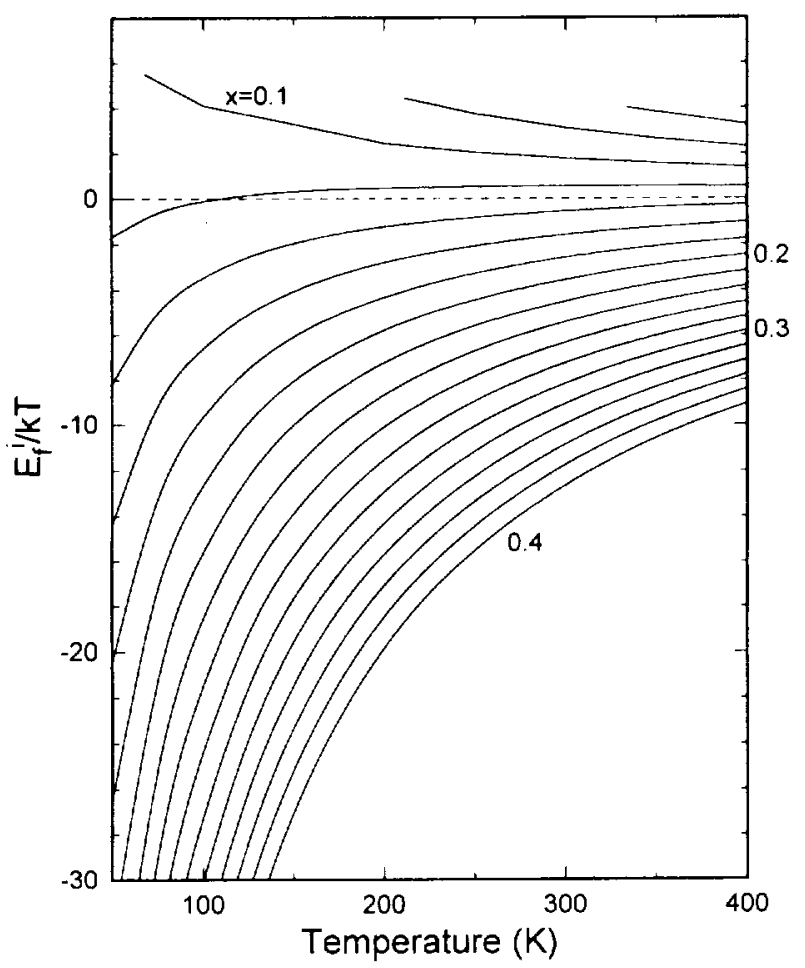

FIG. 2. Calculated reduced Fermi energies for the intrinsic $\mathrm{Hg}_{1}, \mathrm{Zn}_{\mathrm{a}} \mathrm{Te}$ as functions of temperature. The $x$ value is 0.06 for the upper most curve and. with a 0.02 interval, in an ascending order for the other curves from the top to bottom, as indicated by the labels near the curves or on the right margin.
$\Gamma_{6}-\Gamma_{8}$, become negative and $\Gamma_{8}$ becomes the conduction band for these compositions. For $x=0.04$, the band gap stays negative for the entire temperature range of calculation. After the cross over, the effective energy gap remains zero. Figure 3(a) and 3(b) show the calculated intrinsic carrier concentrations and reduced intrinsic Fermi energies, respectively, for those compositions near the band cross over. The curves reflect two competing mechanisms that are present near the band cross over as temperature decreases: (a) decreasing temperature tends to confine the electrons to near the band edge and (b) the closing band gap makes the thermally activated electrons easy to occupy the higher levels in the conduction band. The intrinsic carrier concentrations seem to be not affected by the band cross over indicating that mechanism (a) has the predominant influence on their behavior throughout the entire temperature range. The intrinsic Fermi energies for these compositions are pushed well into the conduction band due to the large density of states in the heavy-mass valence band and $E_{f}^{i} / k T$ keeps increasing with

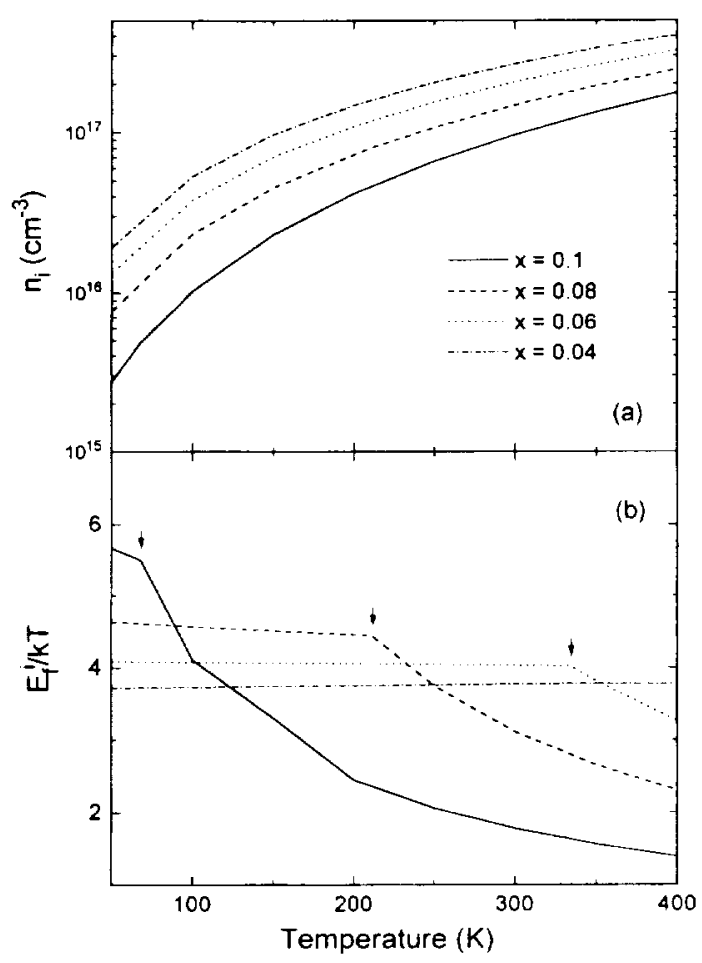

FIG. 3. Calculated intrinsic carrier concentrations (a) and reduced intrinsic Fermi energies (b) as functions of temperature for $\mathrm{Hg}_{1} \quad \mathrm{Zn}, \mathrm{Te}$ with $0.04 \leqslant x \leqslant 0.1$. The arrows in plot $(b)$ indicale the temperatures where the band cross over occurred. 


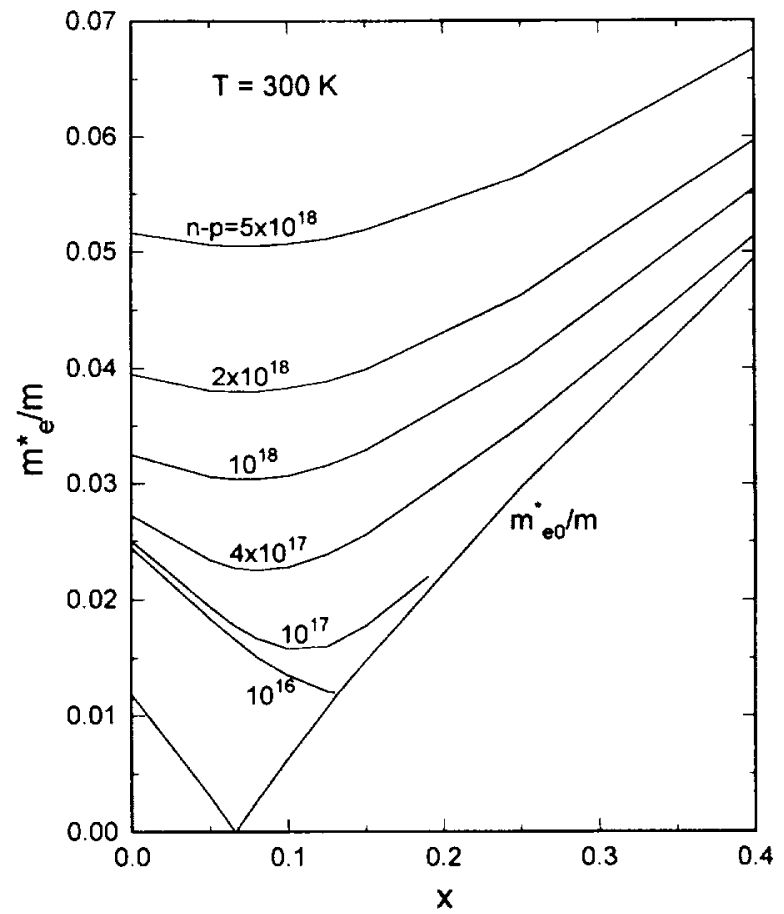

FIG. 4. Calculated transport electron effective masses as functions of alloy composition for $\mathrm{Hg}_{1-\mathrm{x}} \mathrm{Zn}_{\mathrm{r}} \mathrm{Te}$ at $300 \mathrm{~K}$. Each curve is calculated at the Fermi energies corresponding to a fixed $n-p$ value as indicated by the labels. The bottom curve is the band-edge values for the property

decreasing temperature before the band cross over, reflecting a stronger effect of mechanism (b). After the cross over, mechanism (a) comes into play and eventually, by overcoming the effect of mechanism (b), reverses the direction of change. (The actual $E_{f}^{i}$ values decrease.) For $x=0.04$, the Fermi energy decreases with decreasing temperature.

\section{TRANSPORT EFFECTIVE MASS}

In general, the expression for the electron effective mass that appears in the description of transport phenomena is given by $m_{e}^{*}=\hbar^{2} k(d E / d k)^{-1}$ for a given energy band $E(k)$ with arbitrary nonparabolicity. ${ }^{28}$ It is also referred to as susceptibility effective mass ${ }^{22}$ or cyclotron effective mass. ${ }^{29}$ From Eq. (2), we have

$$
\frac{m_{e}^{*}}{m}=\left(1+\frac{m}{\hbar^{2} k} \frac{\partial \varepsilon_{c}}{\partial k}\right)^{-1} .
$$

Józwikowski and Rogalski ${ }^{19}$ adopted a relation for $m_{e}^{*}$ which was derived by Wright et al. ${ }^{22}$ from the Kane model using a series of approximations for the case of semimetals and small carrier concentrations. Among those approximations, the Fermi-Dirac distribution for the conduction band electrons was replaced by a step function, the electrons were treated as occupying all the states inside a sphere in $k$ space, and constant electron levels, $n$, were used instead of the more meaningful $n-p$. In this work, no approximation was made in calculating the effective mass from the Kane model and the Fermi energies that correspond to given $n-p$ values were used in the calculation. For a given energy $E$, Eqs. (1) and (2) were first used to solve for $\varepsilon_{c}$ and $k$, which were subsequently used in evaluating the derivative in Eq. (10).

Figure 4 shows the electron transport effective masses calculated at the Fermi energies corresponding to different levels of the net carrier concentration, $n-p$. Calculations were performed at $T=300 \mathrm{~K}$ and only for those compositions at which the Fermi energy is within the conduction band. To solve the Fermi energies corresponding to a given $n-p=N$ value, the $E_{f}$ in Eqs. (7) and (8) was varied until $|n-p / N-1|<10^{-4}$ was satisfied. Comparing to the results in Ref. 19, one can notice the difference in the shape of the curves, especially for the low net carrier concentrations ( $n$ $-p$ ) on the right-hand side of the band cross over, where the Fermi energies fall below the conduction band edge and the semimetal approximations do not hold. At large net carrier concentrations, where direct comparison can be made (since $n-p \approx n$ for $N \geqslant 10^{18}$ ), the exact calculations given here yield $m_{e}^{*}$ values that are about $7 \%-10 \%$ smaller than those given in Ref. 19. Of particular interest, the electron transport effective mass at the bottom of the conduction band, given by

$$
\frac{m_{e 0}^{*}}{m}=\left(1+\frac{4 m P^{2}}{3 \hbar^{2}} \frac{\Delta+1.5 E_{g}}{E_{g}\left(\Delta+E_{g}\right)}\right)^{-1} \text {, for } E_{g}>0,
$$

and

$$
\frac{m_{e 0}^{*}}{m}=\left(1+\frac{4 m P^{2}}{3 \hbar^{2}\left|E_{g}\right|}\right)^{-1}, \text { for } E_{g}<0,
$$

is also shown in Fig. 4 as a function of composition.

In Ref. 19, the parabolic equivalent density-of-states effective masses were calculated for compositions as low as $x=0.12$ for $50 \leqslant T \leqslant 350 \mathrm{~K}$, where the presumption of nondegenerated semiconductor in deriving the quantity became invalid, rendering the density-of-states effective mass meaningless. It should be noted that the density-of-states effective mass has no inherent physical significance and its main utility is in simplifying the formalism for the computation of $n_{i}$.

\section{SUMMARY}

Intrinsic carrier concentrations, intrinsic Fermi energies, and electron effective mass were calculated for the $\mathrm{Hg}_{1-x} \mathrm{Zn}_{x} \mathrm{Te}$ alloys in the range of $0<x \leqslant 0.4$ and 50 $\mathrm{K} \leqslant T \leqslant 400 \mathrm{~K}$. Unlike previous calculations, no approximation to the band structure, and hence to the density of states, was made beyond those inherent in the Kane $\mathbf{k} \cdot \mathbf{p}$ theory. The Kane secular equation was solved numerically and the exact energy dispersion relation for the conduction band was used in calculating the carrier densities. The previous calculated intrinsic carrier concentrations ${ }^{19}$ are about $10 \%-30 \%$ lower than obtained here. The difference is believed to be a result of the approximation made previously to the energy band structure. The first order derivatives of the energy dispersion relation from the Kane model was derived analytically without any approximation. The calculations included the band cross-over region where band interactions have large effects on the carrier concentrations, Fermi energies, and the effective mass of the alloy system. 


\section{ACKNOWLEDGMENT}

The work was supported by the Microgravity Science and Applications Division of the National Aeronautics and Space Administration.

'A. Sher, A. B. Chen, W. E. Spicer, and C. K. Shih, J. Vac. Sci Technol. A 3, 105 (1985)

${ }^{2}$ E. Cruceanu, D. Niculescu, N. Nistor, and A. Aldea, Rev. Roum. Phys. 9. $5(6)(1964)$.

${ }^{3}$ R. Triboulet, J. Cryst. Growth 86, 79 (1988).

${ }^{7}$ A. Fissel and M. Schenk, Cryst. Res. Technol. 25, 89 (1990).

${ }^{5}$ R. N. Andrews, S. D. Walck, M. W. Price, F. R. Szofran, C.-H. Su, and S L. Lehoczky, J. Cryst. Growth 99, 717 (1990).

${ }^{\circ}$ D. Bagot, R. Granger, and S. Rolland, Phys. Status Solidi B 177, 295 (1993).

${ }^{7}$ R. Triboulet, A. Lasbley, B. Toulouse, and R. Granger, J. Cryst. Growth 79. $695(1986)$.

${ }^{*}$ C.-H. Su, S. L. Lehoczky, and F. R. Szofran, J. Cryst. Growth 86, 87 (1988): 109. $392(1991)$

"Y. G. Sha, C.-H. Su, and S. L. Lehoczky, J. Cryst. Growth (in press)

${ }^{10}$ E. J. Smith, T. Tung, S. Sen, W. H. Konkel, J. B. James, V. B. Harper, B. F. Zuck, and R. A Cole. J. Vac. Sci. Technol. A 5, 3043 (1987).

"A. Sher. A. Tsigelman, E. Weiss, and N. Mainzer, J. Vac. Sci. Technol. A 8. $1093(1990)$

${ }^{12}$ E. A. Patten, M. H. Kalisher, G. R. Chapman, J. M. Fulton, C. Y. Huang,

P. R. Norton, M. Ray, and S. Sen, J. Vac. Sci. Technol. B 9, 1746 (1991).
1.3 J. P. Futurie, J. Cryst. Growth 81. 48.3 (1987).

${ }^{1+}$ J. Piotrowski, K. Adamiec, A. Maciak, and Z. Nowak. Appl. Phys. Lett. 54. 143 (1989).

15. Piotrowski and T. Niedziela. Infrared Phys. 30, 113 (1990).

${ }^{16}$ A. D. Shneider and D. I. Tsiutsiura. Phys. Status Solidi A 5, K39 (1971)

${ }^{17}$ E. Z. Driuha. D. Niculescu, and N. Niculescu. Phys. Status Solidi 29, 813 $(1968)$

${ }^{18}$ B. Toulouse, R. Granger, S. Rolland, and R. Triboulet. J. Phys. IFrancel 48. 247 (1987).

${ }^{19} \mathrm{~K}$. Józwikowski and A. Rogalski, Infrared Phys. 28. 101 (1988).

${ }^{20}$ E. O. Kane, J. Phys. Chem. Solids 1, 249 (1957).

${ }^{21}$ T. C. Harman and A. J. Strauss, J. Appl. Phys. 32. 2265 (1961).

${ }^{22}$ G. B. Wright, A. J. Strauss, and T. C. Harman, Phys. Rev. 125, 1534 (1962).

${ }^{23}$ C.-H. Su, P.-K. Liao, and R. F. Brebrick, J. Electron. Mater. 12. 77 (1983).

${ }^{24}$ W. Abdelhakiem. J. D. Patterson, and S. L. Lehoczky, Mater. Lett. 11, 47 (1991).

${ }^{25} \mathbf{R}$. Dornhaus and G. Nimtz, in Springer Tracts in Modern Physics, edited by G. Höhler (Springer, New York, 1983), Vol. 98.

${ }^{26}$ J. D. Patterson, W. A. Gohbal and S. L. Lehoczky, J. Mater. Res. 7, 2211 (1992)

${ }^{27}$ R. W. Miles, S. Arshed, T. Owens, and R. Hill, Proc, 8th International E. C. Photovoltaic Solar Energy Conference edited by J. Solomon, B. Equer, and P. Helm, 1988 (unpublished), p. 1066.

${ }^{28}$ W. Zawadzki, Adv. Phys. 23, 435 (1974).

${ }^{29}$ J. D. Wiley and R. N. Dexter, Phys. Rev. 181, 1181 (1969). 


